Editorial

Z Gerontol Geriat 2019 · 52:639-640

https://doi.org/10.1007/s00391-019-01636-x

(c) Springer Medizin Verlag $\mathrm{GmbH}$, ein Teil von Springer Nature 2019

\author{
W. Hofmann ${ }^{1,2}$ \\ 'Rechlin/Müritz, Deutschland \\ ${ }^{2}$ Institut für Allgemeinmedizin, Universitätsklinikum Schleswig-Holstein (UkSH), Campus Lübeck, Lübeck, \\ Deutschland
}

\title{
Menschen mit Demenz in der Wohnbevölkerung - gezielt erkennen und passend unterstützen
}

ohne jegliche institutionalisierte Unterstützung. Es führt in gewisser Weise den Diskurs zum bezeichneten Paradigmenwechsel fort, verbunden mit der Aufforderung: „care the carer“!

Die Rostocker Universitätsmedizin und das Deutsche Zentrum für Neurodegenerative Erkrankungen (DZNE) Rostock/Greifswald (Kilimann et al.) evaluieren eine psychotherapeutische Gruppentherapie für Angehörige von $\mathrm{MmD}$, die niedrigschwellig, kompakt und personenorientiert vorgeht. Diese will über Selbsthilfe- und Unterstützungsgruppen hinausgehen, die „nur“ Beratung enthalten. Die kleine Studie ist allerdings negativ. Eine Publikation in diesem Heft ist dennoch ein $\mathrm{zu}$ schätzendes Verdienst - es werden nämlich spezifischer Einzelbedarf bei differenzierten Demenzdiagnosen und die Implementierung im ländlichen Raum fokussiert.

Das Institut für Psychologie der Universität Jena (Theurer et al.) untersucht in einer ebenfalls kleinen Stichprobe erstmals den ansteigenden Anteil (derzeit bei ca. 10\%) der pflegenden (Schwieger-)Söhne. Deren Herangehensweise an die Pflege unterscheidet sich von derjenigen bei (Schwieger-)Töchtern hier in keinster Weise. Das Ergebnis ist interessant und praxisrelevant, denn etablierte Unterstützungssysteme sind nach dieser Pilotstudie gleichermaßen für Söhne und Töchter geeignet, eine Entwicklung gen- dergerechter Programme wäre demnach (?) nicht erforderlich.

Das Deutsche Zentrum für Neurodegenerative Erkrankungen und die universitäre Allgemeinmedizin Greifswald (Monsees et al.) beleuchten die Prävalenz von Demenz bei Migranten. Eine Datenanalyse stellt diese zum einen nach Herkunftsland (bezogen auf ethnische Herkunft und Kontinent) und zum anderen nach Verteilung in den deutschen Bundesländern dar - die Unterschiede sind nicht unbeträchtlich. Die erhobene Gesamtzahl von 5,2\% MmD bei Migranten unterschätzt die „wahre“ Prävalenz höchstwahrscheinlich - verbleibt die Demenz bei Deutschen ohnehin schon unterdiagnostiziert. Wo je nach Herkunftskultur und deutschem Bundesland ein sehr spezifischer Handlungsbedarf besteht: Nun sind hierfür Daten da!

Das Zentrum für Allgemeinmedizin und Geriatrie der Universität Mainz (Wangler et al.) geht davon aus, dass der Hausarzt eine Diagnose der Demenz nicht gerne stellt, sondern lieber an den Facharzt „überstellt“. Dies kontrastiert mit der Forderung nach Frühdiagnostik, die sich $70-80 \%$ der Menschen aller Altersklassen wünschen. Aber gerade der Hausarzt ist nah am Alltag, wo sich frühe Zeichen zuverlässig zeigen. Die Arzthelferin/medizinische Fachangestellte (MFA) könnte Screenings beim Assessment leisten; Tools dürfen delegiert werden. Die kleine, qualitative Befragungsstudie zeigt: Praxispersonal wäre zwar motiviert und sehr wohl bevölkerung befassen, zu Hause lebend - 


\section{Editorial}

reit, eine systematische Organisation mit Ablaufalgorithmus ist aber nicht $\mathrm{zu}$ finden -, sondern eine Forderung für die Zukunft.

Die vier Beiträge wollen Ihre Neugierde wecken, auf Forschungsfragen hinweisen und dazu beitragen, die Versorgung $\mathrm{zu}$ verbessern. Ich hoffe, dass das Lesen Ihnen Freude macht!

Ihr

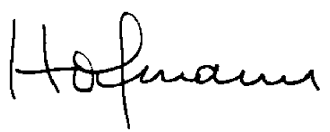

W. Hofmann

\section{Korrespondenzadresse}

\begin{tabular}{ll} 
& $\begin{array}{l}\text { PD Dr. med. W. Hofmann } \\
\text { Institut für Allgemeinmedizin, } \\
\text { Universitätsklinikum } \\
\text { Schleswig-Holstein (UkSH), } \\
\text { Campus Lübeck } \\
\text { Lübeck, Deutschland } \\
\text { werner.hofmann@uksh.de }\end{array}$ \\
\hline Foto:Schrader
\end{tabular}

Interessenkonflikt. W. Hofmann: industriell: Honorare der Fa. Heel $\mathrm{GmbH}$, Baden Baden; institutionell: Präsident Deutsche Gesellschaft für Geriatrie (DGG) e. V. 2010-2012.

\section{Literatur}

1. Statistisches Bundesamt (2018) Pressemitteilung vom 18.12.2018. www.destatis.de. Zugegriffen: 30. Sept. 2019

2. Franke $A$, Kramer $B$, Jann PM, van Holten $K$, Zentgraf A, Otto U, Bischofberger I (2019) Aktuelle Befunde zu "distance caregiving". Was wissen wir und was (noch) nicht? Z Gerontol Geriat 52:521-528. https://doi.org/10.1007/s00391019-01596-2 